\title{
Thermal Imprint Introduced Crystallization of A Solution Processed Subphthalocyanine Thin Film
}

\author{
Yu Shu, Jingfan Wang, Yu Tian, Xiaogan Liang, Shangchao Lin,* and Biwu Ma*
}

The use of organic molecules for thin film electronic devices has been extensively explored over the last decades, with great success realized in organic light emitting diodes, organic photovoltaic cells (OPVs), organic field-effect transistors, and so on. ${ }^{[1]}$ In all of these devices, the morphology and microstructure of organic thin films play critical roles in determining the optical/electronic properties and device characteristics. ${ }^{[2]}$ Generally, thin films with long-range ordered structures, for instance single crystalline thin films, are desired for device applications, considering their higher charge carrier mobility and longer exciton diffusion length than thin films with disordered structures. ${ }^{[3]}$ However, producing organic thin films with long range ordered structures is not trivial, and there are a number of factors affecting the formation of organic thin films, including molecular structures, substrates, processing conditions, etc. Indeed, most single crystalline organic thin films investigated to date suffer from limited size of nanometers or micrometers. ${ }^{[4]}$ Directed assembly of molecular semiconductors to enhance molecular ordering and charge transport has been demonstrated in a variety of systems. ${ }^{[5]}$ However, different material species are introduced into the systems, which could possess negative impacts on the integrity and stability. Posttreatments on disordered organic thin films, including thermal annealing, solvent annealing, solvent-vapor exposure, etc. have shown to be able to introduce molecular reorganization and improve the ordering degree of molecular structures, therefore increasing the intermolecular interactions..$^{[3,6,7]}$ With different ways of posttreatments, amorphous-to-crystalline phase transformation has been observed for a number of polymer and small molecule-based thin films. ${ }^{[7,8]}$

\section{Y. Shu, Prof. B. Ma}

Department of Chemical and Biomedical Engineering Florida State University

95 Chieftan Way, Tallahassee, FL 32306, USA

E-mail:bma@fsu.edu

J. Wang, Prof. S. Lin

Department of Mechanical Engineering

Florida State University

2525 Pottsdamer Street, Tallahassee, FL 32310, USA

E-mail: slin3@fsu.edu

Y. Tian, Prof. S. Lin, Prof. B. Ma

Materials Science and Engineering Program

Florida State University

Tallahassee, FL 32306, USA

Prof. X. Liang

Department of Mechanical Engineering

University of Michigan

2350 Hayward Ave, Ann Arbor, MI 49109, USA

DOI: 10.1002/admi.201600179
Boron subphthlocyanines (SubPcs) are an emerging class of molecular semiconductors with unique optical/electronic properties. ${ }^{[9]}$ The nonplanar pyramid-shaped structure affords SubPcs with high solubility and low crystallinity, allowing for the formation of uniform, grain-boundary-free amorphous thin films via simple solution processing. Our group and others have reported the use of solution processed thin films based on SubPcs in OPVs. ${ }^{[10,11]}$ As compared to high vacuum vapor deposition, solution processing significantly simplifies the fabrication process and reduces the cost. However, the amorphous feature of solution processed SubPc thin films possess relatively low charge carrier mobility, i.e., on the order of $10^{-6} \mathrm{~cm}^{2} \mathrm{~V}^{-1} \mathrm{~s}^{-1}$ much lower than $10^{-3} \mathrm{~cm}^{2} \mathrm{~V}^{-1} \mathrm{~s}^{-1}$ for typical vapor deposited crystalline organic thin films. ${ }^{[12]}$ This amorphous morphology also limits the exciton diffusion length, resulting in OPVs with poor fill factors, low short-circuit currents, and low power-conversion efficiencies. Applying facile posttreatments to convert solution processed amorphous SubPc thin films into highly crystalline ones is of great interest. However, neither thermal annealing nor solvent annealing is able to effectively tune the morphology of solution processed SubPc films.

Herein, we report a facile posttreatment approach capable of introducing molecular ordering (or crystallization) in solution processed organic semiconductor thin films through a thermal imprint process (or thermal annealing under mechanical gauge pressure). This work follows our previous study, in which we have demonstrated that thermal nanoimprint of a solution processed amorphous thin film of 2-allylphenoxy(subphthalocyaninato)boron(III) (SubPc-A), could generate stable nanoscale crystalline domains. ${ }^{[13]}$ In this work, we show that large area phase change from amorphous to crystalline morphology for solution processed SubPc-A thin films can be realized by thermal imprint with a flat mold. Using optical microscope, X-ray diffraction, atomic force microscope (AFM), and scanning electron microscope (SEM), we have characterized the morphological properties of SubPc-A films upon different posttreatments. The morphological change induced by thermal imprint results in enhanced electronic properties, in particular the hole mobility that increases from $\approx 1 \times 10^{-7}$ to $\approx 2 \times 10^{-3} \mathrm{~cm}^{2} \mathrm{~V}^{-1} \mathrm{~s}^{-1}$. This change of morphological and electronic properties could not be achieved by either thermal annealing or room temperature imprint alone. We have also applied molecular dynamics (MD) simulations to investigate the molecular ordering and stability of the SubPc-A crystal under different geometrical confinement and temperature conditions. This simulation result clearly indicates that both high temperature (via thermal annealing) and geometrical confinement (via imprinting) are necessary for the resulting 
www.advmatinterfaces.de
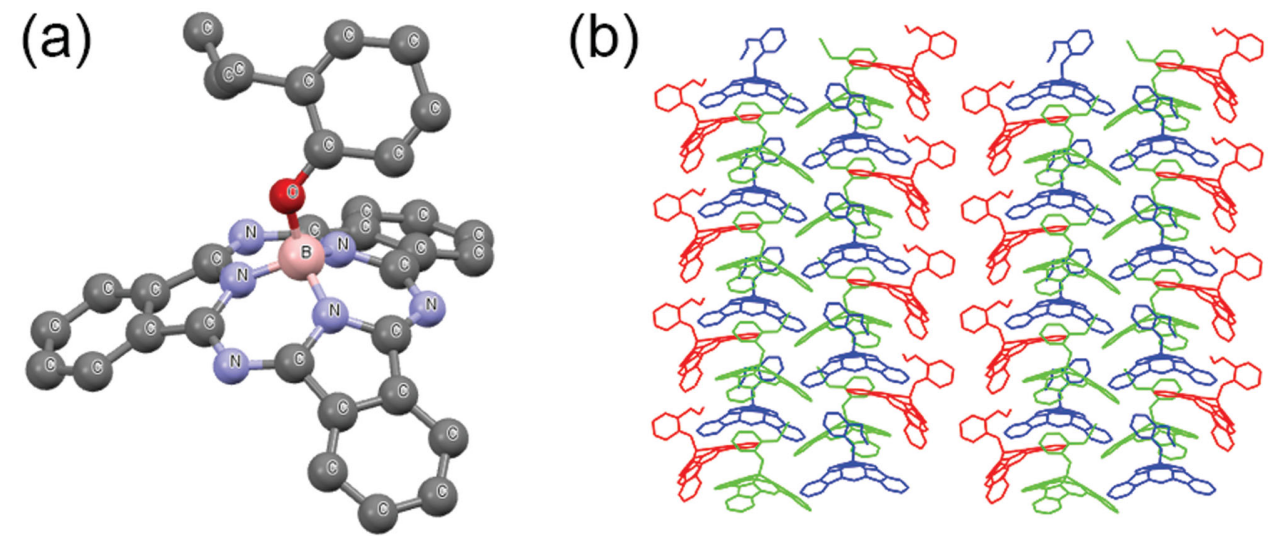

Figure 1. a) The molecular structure of SubPc-A and b) its single crystal structure.

facile molecular ordering in the SubPc-A thin film. This implication is highly consistent with our experimental observations.

Figure 1 shows the molecular structure of SubPc-A and its molecular stacking in single crystals. The columnar stacking of the macrocycles as a result of the cone-shaped geometry is significantly different from other axially functionalized SubPcs with molecular packing along axial groups. ${ }^{[11,14]}$ Due to the strong interaction among the delocalized $\pi$ molecular orbitals of the macrocycles, this columnar stacking is likely to possess a high charge transport efficiency. In other words, achieving highly crystalline SubPc-A thin films represents an effective approach to enhancing the carrier mobility.

Amorphous SubPc-A thin films were prepared by spin coating chloroform solutions of SubPc-A on top of poly(3,4ethylenedioxythiophene)-poly(styrenesulfonate)(PEDOT:PSS)/ indium tin oxide (ITO)/glass substrates (See the Experimental Section). We investigated different posttreatment methods for the thin film morphology control/modulation, including thermal annealing, imprint at room temperature, and thermal imprint. $90{ }^{\circ} \mathrm{C}$ was chosen for thermal annealing and thermal imprint, as it was previously identified as the optimal temperature for nanoimprint processes, which can effectively introduce nanoscale molecular stacking. ${ }^{[13]}$ All the posttreatments were conducted for a variety of durations, and the results were compared to the as-cast amorphous thin films. We found that only thermal imprint processed samples exhibit significant change of thin film morphology. Figure 2 shows the optical microscopy images acquired under normal and polarized light illuminations for the solution processed SubPcA thin films upon different posttreatments. Figure 2a,c,e under normal light have almost identical features, so do Figure $2 \mathrm{~b}, \mathrm{~d}$,f under polarized light (totally dark due to the lack of crystalized structure), suggesting that the smooth amorphous morphology remained mainly unchanged after thermal annealing at $90{ }^{\circ} \mathrm{C}$ for $12 \mathrm{~h}$ and imprint at room temperature for $12 \mathrm{~h}$. Significantly different morphology over large area from crystalized structure with little defects was observed for the SubPc-A thin films after thermal imprint at $90{ }^{\circ} \mathrm{C}$ for $12 \mathrm{~h}$, as shown in Figure 2g,h under normal light and polarized light, respectively. Overall, the amorphous SubPc-A thin films have been fully converted into highly crystalline thin films after thermal imprint $90^{\circ} \mathrm{C}$ for $12 \mathrm{~h}$. It should be pointed out that both thermal annealing and imprint were performed in an oven under nitrogen environment, suggesting neither
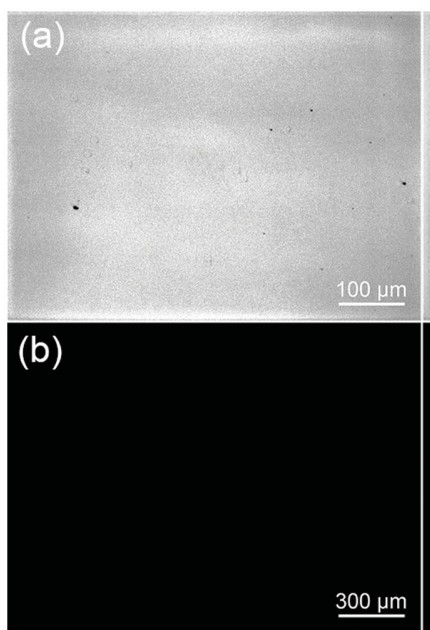

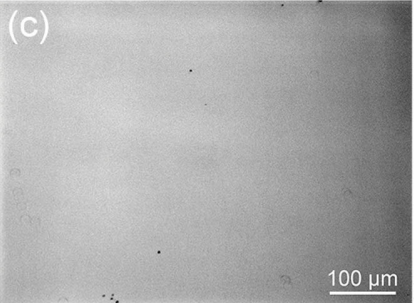

(d)

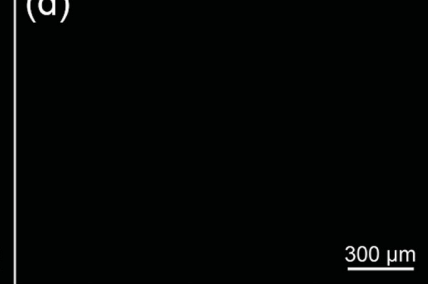

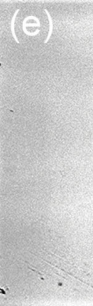

(f)

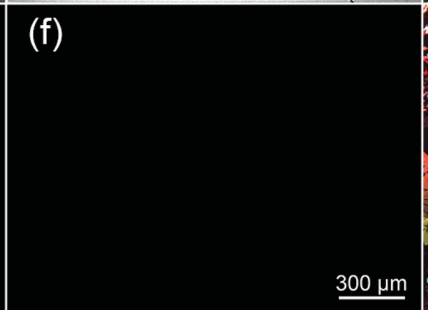

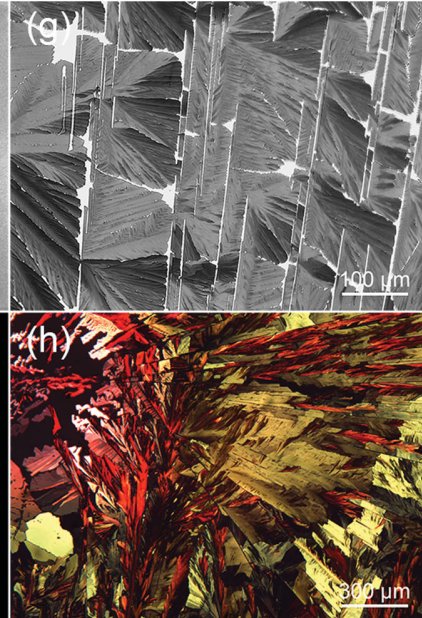

Figure 2. Optical images of the SubPc-A films upon different treatments under normal (top) and polarized light (bottom): a,b) as spun cast, c,d) thermal annealing at $90^{\circ} \mathrm{C}$ for $12 \mathrm{~h}, \mathrm{e}, \mathrm{f}$ ) imprint at room temperature for $12 \mathrm{~h}, \mathrm{~g}, \mathrm{~h}$ ) thermal imprint at $90^{\circ} \mathrm{C}$ for $12 \mathrm{~h}$. 




Figure 3. XRD patterns of SubPc-A thin films with different treatments.

heating method nor environment would influence the amorhpous to crystalline phase change.

X-ray diffraction (XRD) was used to characterize the crystallinity of SubPc-A films upon different treatments. As shown in Figure 3, no diffraction peaks are observed for the as-cast thin film and the thin films after thermal annealing or imprint at room temperature alone, indicating a negligible degree of crystallinity. However, the XRD spectra of the thin films upon thermal imprint at $90{ }^{\circ} \mathrm{C}$ for $12 \mathrm{~h}$ displays very clear peaks associated with high degree of ordered molecular stacking (or crystallization). A powder diffraction program was simulated using the single crystal structure for SubPc-A. By calculating the $d$-spacing from Bragg's law, the $10^{\circ}$ peak is indexed as (003) and the $15^{\circ}$ peak is indexed as (104).

We also used SEM and AFM to further characterize the thin film microstructures. Figure 4 shows SEM and AFM images of SubPc-A thin films under different treatments. Consistent with optical microscopy images, we observed amorphous morphology with extremely high surface smoothness (root mean squared roughness $R_{\mathrm{q}}<1 \mathrm{~nm}$ ) for as-cast, thermally annealed, and room temperature imprinted thin films. For the thermally imprinted thin films in Figure 4g, gaps and pinholes were observed in SEM images. These defects, making the thin films less smooth and uniform, were introduced during the amorphous to crystalline phase transfer process because of the change of specific volume and density. AFM provided morphological features with higher resolution than SEM. The size of AFM image in Figure $4 \mathrm{~h}$ is the same as the small square in Figure $4 \mathrm{~g}$ with a $5 \mu \mathrm{m} \times 5 \mu \mathrm{m}$ area. From SEM images Figure 4a,c,e, we cannot see apparent difference at this scale (scale bar $=40 \mu \mathrm{m}$ ) among samples from three treatment condition-as spin cast, thermally annealing, and room temperature imprinting, in another word, those samples did not crystallize. Although from AFM images Figure 4b,d,h, we can see slight difference, they all have a low $R_{\mathrm{q}}$ value. In AFM image Figure $4 \mathrm{~h}$, clear crystalline domains with size of micrometers and grain boundaries were observed. The crystalline domains have extremely high surface smoothness with roughness similar to the amorphous thin films $\left(R_{\mathrm{q}}<1 \mathrm{~nm}\right)$.

The change of moprhology and microstructure of organic semiconductor thin films usually has direct impact on the electronic properties, for instance, charge transport. The hole carrier mobilities of SubPc-A thin films upon different treaments were measured by space charge limited current (SCLC) method, in a device structure of ITO/PEDOT:PSS $(30 \mathrm{~nm}) /$ SubPc-A $(150 \mathrm{~nm}) / \mathrm{Au}(55 \mathrm{~nm})$ as illustrated in the inset of Figure 5. The $J-V$ curves shown in Figure 5 could be well fitted with the SCLC model, and the corresponding hole mobility values are extracted to be $0.71 \times 10^{-7}$ (as-cast), $1.24 \times 10^{-7}$ (thermally annealed), $0.96 \times 10^{-7}$ (room temperature imprinted), and $1.79 \times 10^{-3} \mathrm{~cm}^{2} \mathrm{~V}^{-1} \mathrm{~s}^{-1}$ (thermally imprinted), for the
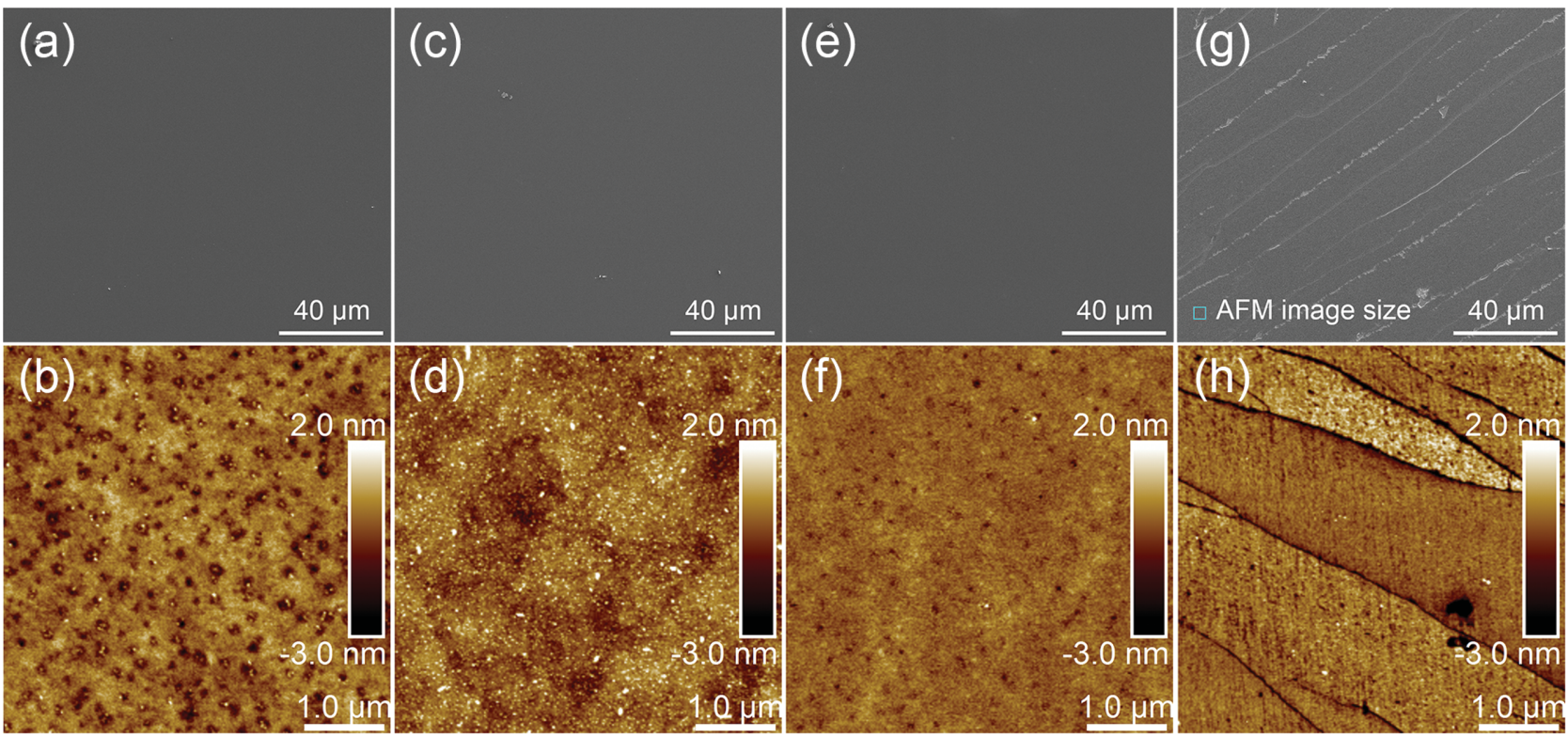

Figure 4. SEM (top) and AFM (bottom) images of SubPc-A thin films after different treatments: a,b) as cast film, c,d) thermally annealed film, $e, f)$ room temperature imprinted film, g,h) thermally imprinted film. 


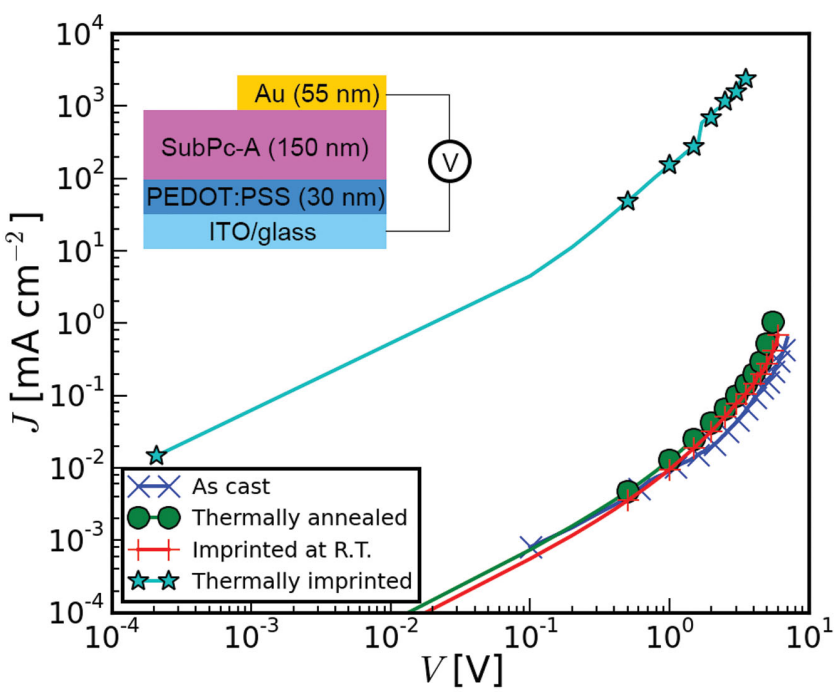

Figure 5. $J-V$ curves for the space charge limited current (SCLC) measurement of the hole carrier mobilities of SubPc-A thin films upon different treatments. The inset shows the device structure.

SubPc-A thin films with different treatments. These results, for the first time, experimentally demonstrate the expected thermal-imprint-induced enhancement of the hole mobility of SubPc films and also strongly implies that such mobility enhancement is attributed to the morphological change from amorphous to crystalline.

It is still not practical to simulate the entire crystallization process (takes minutes to complete) of amorphous molecular systems using classical MD simulations (can cover $<100 \mathrm{~ns}$ ). ${ }^{[15]}$ Therefore, we used MD simulations here to investigate the reverse process with respect to crystallization, focusing on the structural stability and degree of ordering of single SubPc-A crystal, under various temperatures $(200,400$, and $600 \mathrm{~K})$, on a layer of $\mathrm{SiO}_{2}$ that mimics the surface of the flat oxidized silicon template. Note that a more stable and ordered final crystal structure (with lower energy) usually implies faster crystallization due to the larger thermodynamic driving force (the energy difference between the initial amorphous structure and the final crystalline structure). Two systems were considered here: one with the $\mathrm{SiO}_{2}$ layer (together with its periodic image) compressing both the top and the bottom surfaces of the crystal to mimic the imprinting process (Figure 6a), and the other with the top surface of the crystal exposed to vacuum without any confinement (Figure 6b). The degrees of molecular ordering of SubPc-A were measured by the angular distribution of the SubPc-A molecules with respect to the direction ( $z$-axis) normal to the $\mathrm{SiO}_{2}$ surface (see Figure 7a,b). For SubPc-A molecules facing up, the angle $\alpha=0^{\circ}-90^{\circ}$, while for the ones facing down, $\alpha=90^{\circ}-180^{\circ}$. For bulk SubPc-A crystals (the middle layer in Figure 6) without interfering with the $\mathrm{SiO}_{2}$ template, primary peaks, located at $30^{\circ}-50^{\circ}$ (facing up) and $140^{\circ}-160^{\circ}$ (facing down), were observed under all the temperature values considered (middle panels in Figure $7 \mathrm{c}, \mathrm{d}$ ).

In general, from MD simulations we can observe that increasing the temperature from 200 to $600 \mathrm{~K}$ introduces small perturbation to the single crystal structure of SubPc-A with imprinting (reflected by slight broadening of the angle distribution in Figure $7 \mathrm{c}$ ), while such perturbation is greatly amplified

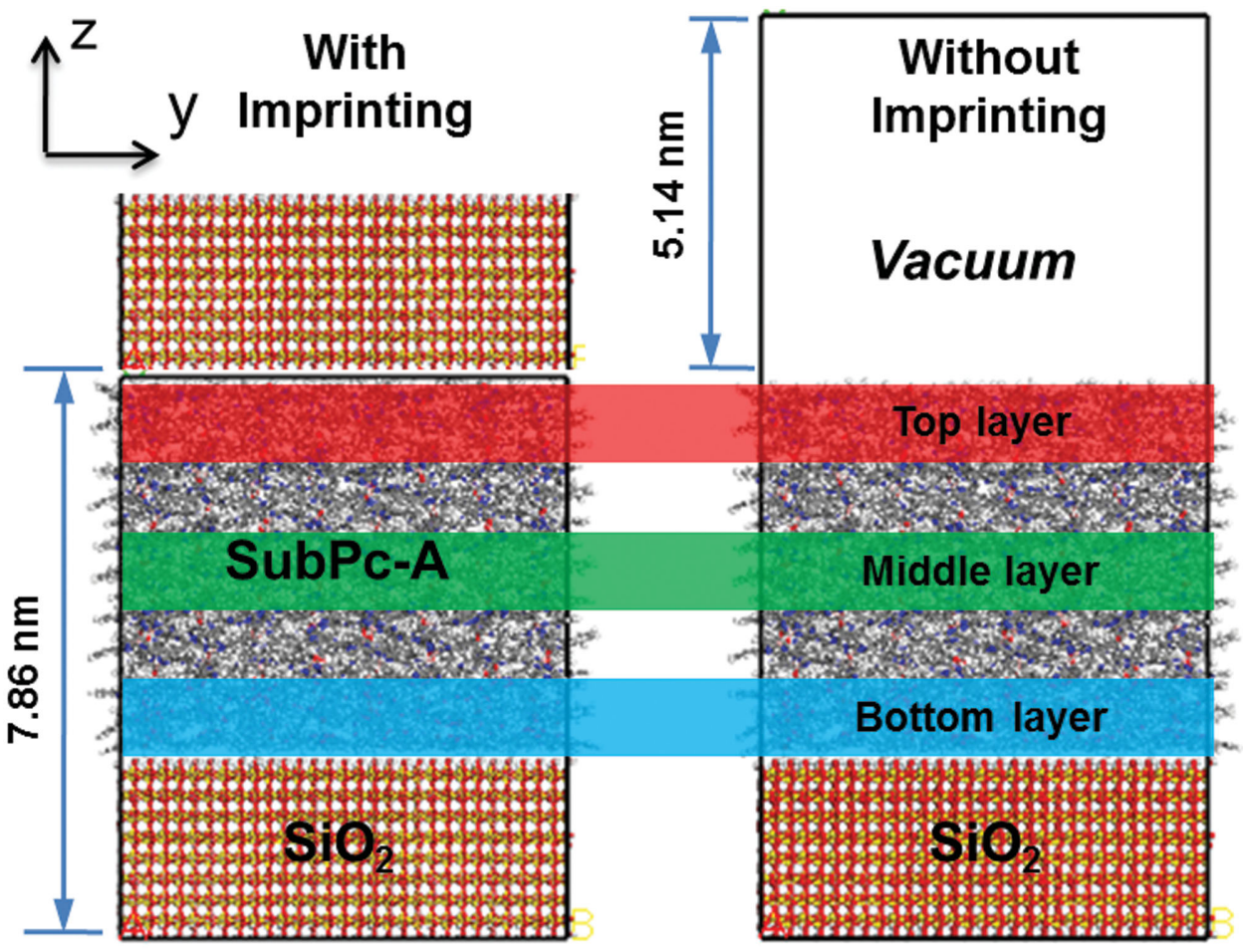

Figure 6. MD simulated configurations for single SubPc-A crystal on a flat $\mathrm{SiO}_{2}$ template: a) with imprinting (confinement on both the top and bottom surfaces, achieved via the periodic image of $\mathrm{SiO}_{2}$ ) and b) without imprinting (no confinement on the top surface). The crystal was divided into three layers (top, middle, and bottom) for subsequent data analyses in Figure 7. 
a)

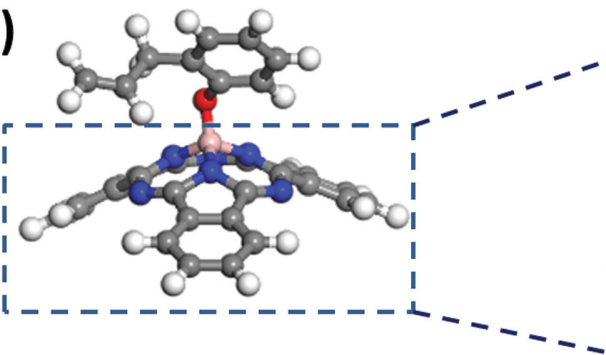



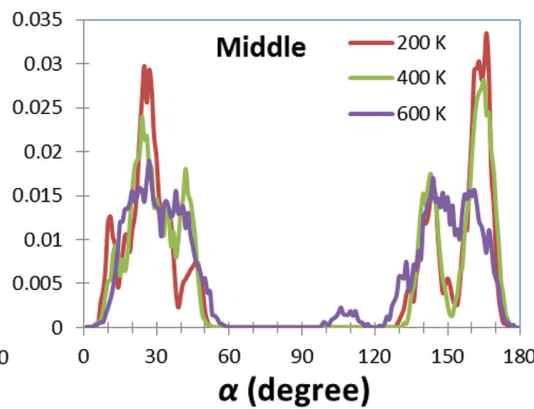

b)

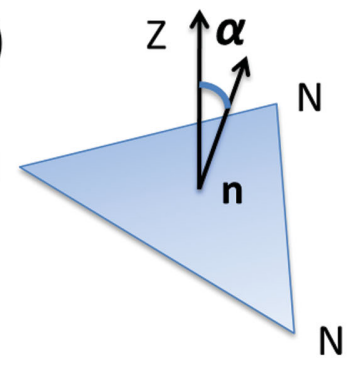


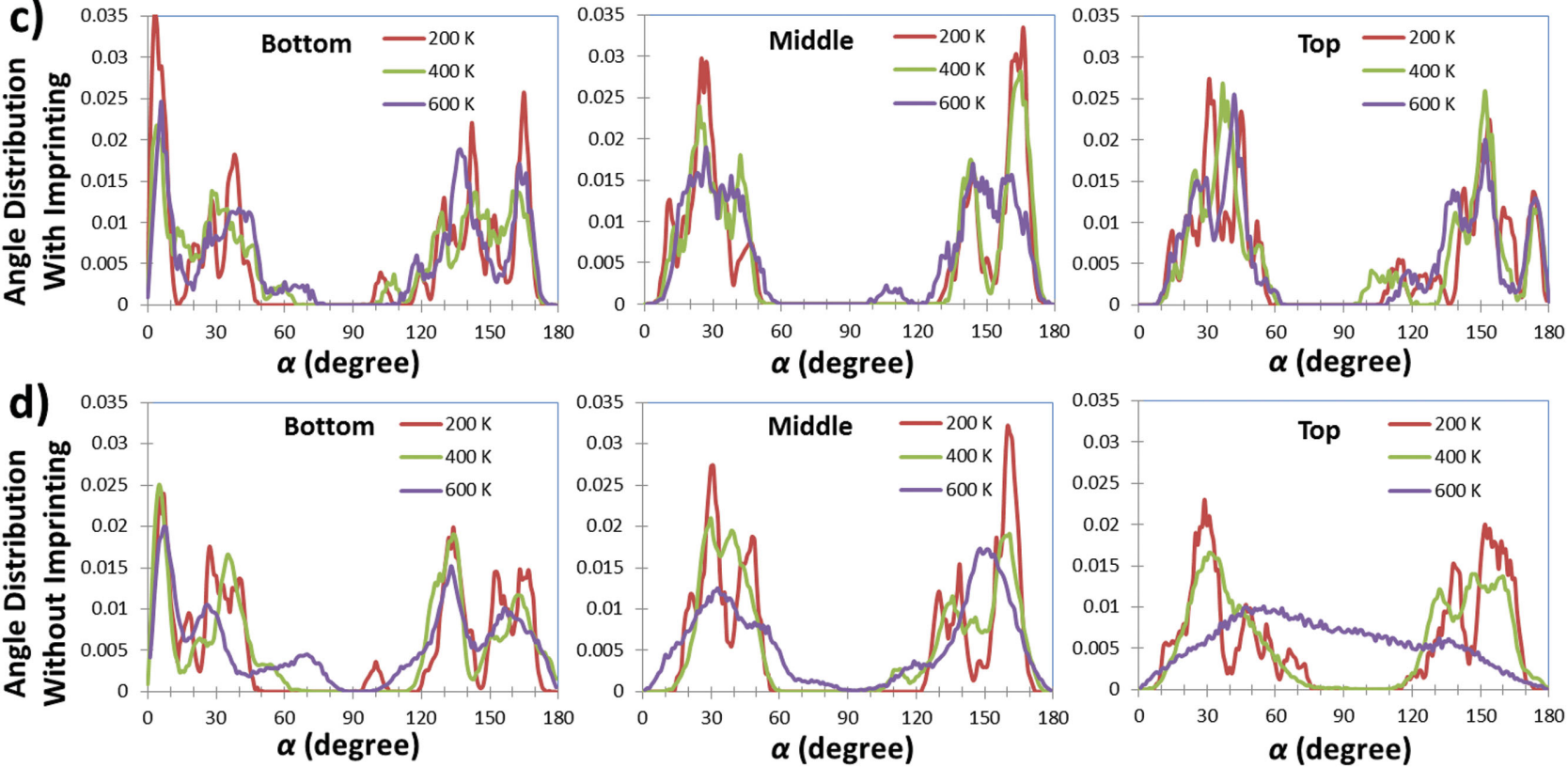

Figure 7. a) Definition of the principal triangular plane of the SubPc-A molecule, connected by the three $N$ atoms. b) Definition of the principal axis normal to the plane, $n$, and the angle formed between the principal axis and the $z$-axis normal to the $\mathrm{SiO}_{2}$ template, $\alpha$. Probability distribution of $\alpha$ is used here as a descriptor to quantify the degrees of molecular ordering of SubPc-A. c) Distribution of $\alpha$ at various temperatures with imprinting, corresponding to the system shown in Figure 6a. d) Distribution of $\alpha$ at various temperatures without imprinting, corresponding to the system shown in Figure $6 b$.

without imprinting (Figure 7d). Specifically, the top SubPc-A layer with geometrical confinement remains ordered even at $600 \mathrm{~K}$ (right panel in Figure 7c), while the top SubPc-A layer exposed to vacuum is completely disordered at $600 \mathrm{~K}$ (smearing of the peaks, right panel in Figure 7d). Interestingly, we found that the various pressure values considered here (1-100 bars) have negligible effects on the angle distribution results, indicating that geometrical confinement alone, without significant pressurization, is enough to introduce the fast molecular ordering and crystallization observed experimentally. Geometrical confinement via the $\mathrm{SiO}_{2}$ template also introduces new peaks $\left(5^{\circ}\right.$ for the bottom layers with or without imprinting, and $175^{\circ}$ for the top layer with imprinting) in angle distributions (see the left and right panels of Figure 7c,d), reflecting small molecular reorientation of SubPc-A on the $\mathrm{SiO}_{2}$ template due to such confinement. The above computational results are consistent with the trend observed experimentally on the role of geometrical confinement (imprinting) on the crystal stability and molecular ordering of the SubPc-A thin film.

On the other hand, too strong geometrical confinement can lead to interlocked or jammed SubPc-A molecules, resulting in slow molecular diffusivity and high energy barriers for molecular rearrangement during crystallization. Therefore, thermal annealing is a necessary process to elevate the kinetic energy of SubPc-A molecules so that they can escape the local energy minima due to jamming in the glassy state. In our simulation results, the $200 \mathrm{~K}$ case exhibits perfect crystallinity but no flexibility for molecular rearrangement and very low molecular diffusivity, while the $400 \mathrm{~K}$ case (close to the annealing temperature of $90{ }^{\circ} \mathrm{C}$ used experimentally) starts to exhibit some flexibility (small broadening of the angle distribution peaks) and higher molecular diffusivity. The computational results again confirm the experimentally observed role of the thermal annealing process on the kinetically driven growth of the SubPc-A thin film.

In summary, we demonstrate a new simple and scalable method, thermal-imprint, to convert amorphous organic semiconductor thin films to crystalline ones. With the amorphous to crystalline transformation, the hole carrier mobility of SubPc-A films show a dramatic enhancement of over a few orders of magnitude. An in-depth understanding of the underlying science associated with this thermal imprint-induced 
morphological change in SubPc-A films has been achieved by the MD simulations. Our work suggests that thermal imprint is a highly promising posttreatment method to achieve high performance organic electronic films. Further studies of the crystallization kinetics are under way. Ongoing work also involves the investigation of the effect of morphological change on the device performance of organic solar cells based on SubPc-A, as well as applying thermal imprint on other molecular systems for morphology control.

\section{Experimental Section}

Materials: ITO coated glass substrates were purchased from thin film devices. PEDOT:PSS (Baytron-PH500) was purchased from H. C. Starck. Gold shot (99.99+\%) and chloroform (99+\%, anhydrous) were purchased from Sigma-Aldrich. SubPc-A was synthesized as reported in a previous paper. ${ }^{[15]}$ Single crystals of SubPc-A were grown by slow diffusion of methanol into dichloromethane solutions of SubPc-A.

SubPc-A Thin Film Preparation: ITO glass substrates were cleaned by ultrasonication in soapy water, acetone, isopropanol, and deionized water for $15 \mathrm{~min}$ each; blown dry with nitrogen gas; and then treated with UV ozone for $20 \mathrm{~min}$. The PEDOT:PSS solution was passed through a $0.45 \mu \mathrm{m}$ polyvinylidene fluoride syringe filter before spin coating at $3500 \mathrm{rpm}$ for $30 \mathrm{~s}$. Then the substrates with PEDOT:PSS were heated at $140^{\circ} \mathrm{C}$ in air for $20 \mathrm{~min}$. The SubPc-A solution in chloroform was passed through a $0.20 \mu \mathrm{m}$ polytetrafluoroethylene filter before spin coating at $1500 \mathrm{rpm}$ for $40 \mathrm{~s}$. The thickness of SubPc-A layer was controlled by concentration.

Thermal Imprint Process: The imprint tool and imprinting scheme (Scheme 1) are shown as below. The assembled structure to be pressed was stacked in the following order from bottom to top: a silicon wafer with mold release agent face up, imprinted sample face down, and a polytetrafluoroethylene slice. The pressure was estimated at 200-500 psi. A Thermo Scientific furnace FB1315M with temperature controller was used to get specific temperature. Samples for thermally annealing (only heating) and thermally imprint (heating and pressure) were heated at $90{ }^{\circ} \mathrm{C}$ for $12 \mathrm{~h}^{\left[{ }^{[13]}\right.}$ After the process, the samples were cooled at room temperature. Once the tool reached room temperature, pressure was released and the template was removed from the substrate.

Characterizations: X-ray diffraction (XRD) spectroscopy was performed with a Panalytical X'PERT Pro powder X-Ray diffractometer with a $\mathrm{Cu}$ $X$-ray source. Atomic force microscopy was performed with a Bruker Dimension Icon in tapping mode with an OTESPA-R3 tip.

Hole mobility characterization for the SubPc-A was measured in the device structure of ITO/PEDOT:PSS $(30 \mathrm{~nm}) / \mathrm{SubPc}-\mathrm{A}(150 \mathrm{~nm}) /$ $\mathrm{Au}(55 \mathrm{~nm})$. A gold electrode was used to ensure a hole-only device, which was deposited in a high vacuum chamber (under $1 \times 10^{-6} \mathrm{mbar}$ ). The current density-voltage $(U-V)$ characteristics were recorded with a Keithley 2400. The results were linearly fitted with the SCLC model as Equation (1)


Scheme 1. Setup for thermal imprint.
$J=\frac{9}{8} \varepsilon_{0} \varepsilon \mu \frac{1}{L^{3}} V^{2}$

where $J$ is measured current density in $\mathrm{A} \mathrm{cm}^{-2}, \varepsilon_{0}$ is the vacuum permittivity $8.85 \times 10^{-12} \mathrm{~A} \mathrm{~s} \mathrm{~V}^{-1} \mathrm{~m}^{-1}, \varepsilon$ is dielectric permittivity constant (assumed to be 3 for organic semiconductors), $\mu$ is hole mobility in $\mathrm{cm}^{2} \mathrm{~V}^{-1} \mathrm{~s}^{-1}, L$ is the film thickness in $\mathrm{cm}$, and $V$ is the applied voltage in $V$. Treatment duration for hole mobility measurements was 30 min.

Molecular Dynamics Simulations: MD simulations of the single SubPc-A crystal on a layer of $\mathrm{SiO}_{2}$ (or $\alpha$-quartz, to mimic the oxidized surface of the flat silicon template) were carried out using the GROMACS 4.5.7 package. ${ }^{16]}$ The initial configuration of the single SubPc-A crystal was obtained via the XRD powder diffractogram ${ }^{[17]}$ and that of the $\mathrm{SiO}_{2}$ template was obtained from existing crystal databases. Both systems shown in Figure 6 underwent energy minimizations using the conjugate gradient method before further equilibration. 3D periodic boundary conditions were applied for all the systems. The system with imprinting (Figure 6a) was equilibrated under the NPT ensemble (at 200,400 , and $600 \mathrm{~K}$, and various pressures ranging from 1 to 100 bars) for 25 ns. The system without imprinting (Figure 6b) was equilibrated under the NVT ensemble (at 200,400, and $600 \mathrm{~K}$ ) with vacuum on top of the SubPc-A layer for $25 \mathrm{~ns}$. The last $10 \mathrm{~ns}$ from both systems were collected for data analysis as shown in Figure 7 (angle distributions). The Nosé-Hoover temperature coupling ${ }^{[18]}$ and the Parrinello-Rahman pressure coupling ${ }^{[19]}$ methods were utilized to control temperatures and pressures. The SubPc-A molecule and the $\mathrm{SiO}_{2}$ template were modeled using the universal force field, ${ }^{[20]}$ and the partial atomic charges of SubPc-A were obtained quantum-mechanically using the semiempirical method $\mathrm{PM}^{[21]}$ implemented in program MOPAC7.1. Long-range electrostatic interactions were treated using the particle mesh Ewald summation method. ${ }^{[22]} A$ cutoff distance of $1 \mathrm{~nm}$ was used for the nonbonded Lennard-Jones and electrostatic potentials. A time step of $0.5 \mathrm{fs}$ was used to integrate the dynamic equations of motion.

\section{Acknowledgements}

The authors thank the Florida State University for financial support through the university Energy and Materials Initiative. The authors also acknowledge Plengchart Prommapan from Dr. David Van Winkle's group (Department of Physics, FSU) and Junfei Xia from Dr. Jingjiao Guan's group (Department of Chemical \& Biomedical Engineering, FSU) for helping with optical microscope characterization, as well as Dr. Eric Lochner from the Condensed Matter and Material Physics (CMMP) at FSU for providing access to SEM and AFM.

Received: March 1, 2016 Revised: May 10, 2016 Published online: June 22, 2016

[1] S. R. Forrest, Nature 2004, 428, 911.

[2] a) V. Coropceanu, J. Cornil, D. A. da Silva Filho, Y. Olivier, R. Silbey, J.-L. Brédas, Chem. Rev. 2007, 107, 926; b) A. A. Virkar, S. Mannsfeld, Z. Bao, N. Stingelin, Adv. Mater. 2010, 22, 3857; c) R. Li, W. Hu, Y. Liu, D. Zhu, Acc. Chem. Res. 2010, 43, 529.

[3] R. R. Lunt, J. B. Benziger, S. R. Forrest, Adv. Mater. 2010, 22, 1233.

[4] L. Jiang, H. L. Dong, W. P. Hu, J. Mater. Chem. 2010, 20, 4994.

[5] a) Y. Zhang, D. Hanifi, E. Lim, S. Chourou, S. Alvarez, A. Pun, A. Hexemer, B. W. Ma, Y. Liu, Adv. Mater. 2014, 26, 1223; b) M. J. Hollamby, M. Karny, P. H. H. Bomans, N. A. J. M. Sommerdijk, A. Saeki, S. Seki, H. Minamikawa, I. Grillo, B. R. Pauw, P. Brown, J. Eastoe, H. Mohwald, T. Nakanishi, Nat. Chem. 2014, 6, 941; c) B. J. Rancatore, C. E. Mauldin, S. H. Tung, C. Wang, A. Hexemer, J. Strzalka, J. M. J. Frechet, T. Xu, ACS Nano 2010, 4, 2721. 
[6] a) D. J. Mascaro, M. E. Thompson, H. I. Smith, V. Bulović, Org. Electron. 2005, 6, 211; b) P. Vlachos, B. Mansoor, M. P. Aldred, M. O'Neill, S. M. Kelly, Chem. Commun. 2005, 23, 2921; c) J. Rivnay, L. H. Jimison, J. E. Northrup, M. F. Toney, R. Noriega, S. Lu, T. J. Marks, A. Facchetti, A. Salleo, Nat. Mater. 2009, 8, 952; d) J. Zhang, I. Salzmann, P. Schäfer, M. Oehzelt, S. Duhm, J. P. Rabe, N. Koch, J. Mater. Res. 2009, 24, 1492; e) S.-W. Park, J.-M. Choi, K. H. Lee, H. W. Yeom, S. Im, Y. K. Lee, J. Phys. Chem. B 2010, 114, 5661; f) J. Smith, R. Hamilton, Y. Qi, A. Kahn, D. D. C. Bradley, M. Heeney, I. McCulloch, T. D. Anthopoulos, Adv. Funct. Mater. 2010, 20, 2330.

[7] Y. Diao, L. Shaw, Z. A. Bao, S. C. B. Mannsfeld, Energy Environ. Sci. 2014, 7, 2145.

[8] a) Z. Hu, G. Baralia, V. Bayot, J.-F. Gohy, A. M. Jonas, Nano Lett. 2005, 5, 1738; b) B. C. Okerberg, C. L. Soles, J. F. Douglas, H. W. Ro, A. Karim, D. R. Hines, Macromolecules 2007, 40, 2968.

[9] a) G. E. Morse, T. P. Bender, ACS Appl. Mater. Interfaces 2012, 4, 5055; b) C. G. Claessens, D. Gonzalez-Rodriguez, T. Torres, Chem. Rev. 2002, 102, 835.

[10] a) B. Ma, C. H. Woo, Y. Miyamoto, J. M. J. Fréchet, Chem. Mater. 2009, 21, 1413; b) B. W. Ma, Y. Miyamoto, C. H. Woo, J. M. J. Frechet, F. Zhang, Y. Liu, Org. Photovoltaics X 2009, 7416, $74161 \mathrm{E}$.

[11] C. E. Mauldin, C. Piliego, D. Poulsen, D. A. Unruh, C. Woo, B. Ma, J. L. Mynar, J. M. J. Fréchet, ACS Appl. Mater. Interfaces 2010, 2, 2833.
[12] a) J. Xue, S. Uchida, B. P. Rand, S. R. Forrest, Appl. Phys. Lett. 2004, 84, 3013; b) M. D. Perez, C. Borek, S. R. Forrest, M. E. Thompson, J. Am. Chem. Soc. 2009, 131, 9281.

[13] X. Liang, T. Chen, Y.-S. Jung, Y. Miyamoto, G. Han, S. Cabrini, B. Ma, D. L. Olynick, ACS Nano 2010, 4, 2627.

[14] a) F. Camerel, G. Ulrich, P. Retailleau, R. Ziessel, Angew. Chem. Int Ed. 2008, 47, 8876; b) Y. Ren, A. M. Hiszpanski, Y. L. Loo, Chem. Mater. 2015, 27, 4008.

[15] a) A. Laio, M. Parrinello, Proc. Natl. Acad. Sci. USA 2002, 99, 12562; b) M. R. Sorensen, A. F. Voter, J. Chem. Phys. 2000, 112, 9599.

[16] B. Hess, C. Kutzner, D. van der Spoel, E. Lindahl, J. Chem. Theory Comput. 2008, 4, 435.

[17] X. Liang, T. Chen, Y.-S. Jung, Y. Miyamoto, G. Han, S. Cabrini, B. Ma, D. L. Olynick, ACS Nano 2010, 4, 2627.

[18] a) S. Nosé, J. Chem. Phys. 1984, 81, 511; b) W. G. Hoover, Phys. Rev. A 1985, 31, 1695

[19] M. Parrinello, A. Rahman, J. Appl. Phys. 1981, 52, 7182.

[20] A. K. Rappe, C. J. Casewit, K. S. Colwell, W. A. Goddard, W. M. Skiff, J. Am. Chem. Soc. 1992, 114, 10024.

[21] a) J. J. P. Stewart, J. Comput. Chem. 1989, 10, 209; b) J. J. P. Stewart, J. Comput. Chem. 1989, 10, 221; c) J. J. P. Stewart, J. Comput. Chem. 1991, 12, 320; d) J. J. P. Stewart, J. Mol. Model. 2004, 10, 155.

[22] a) U. Essmann, L. Perera, M. L. Berkowitz, T. Darden, H. Lee, L. G. Pedersen, J. Chem. Phys. 1995, 103, 8577; b) T. Darden, D. York, L. Pedersen, J. Chem. Phys. 1993, 98, 10089. 